\title{
Application and methodological improvements to the floating-wire technique to characterize the magnetic properties of a spectrometer dipole
}

\author{
H. J. Stein ${ }^{\text {a) }}$ and G. Krol \\ Institut für Kernphysik, Forschungszentrum Jülich, 52425 Jülich, Germany \\ S. Barsov and V. Koptev \\ High Energy Physics Department, Petersburg Nuclear Physics Institute, 188350 Gatchina, Russia
}

(Received 26 September 2000; accepted for publication 26 January 2001)

\begin{abstract}
The floating wire technique was applied to characterize the focusing properties of the D2 spectrometer magnet to be used in the ANKE installation of the COSY Jülich accelerator facility. Trajectories corresponding to 140-600 MeV/c ejectiles emerging from a target in front of the rectangular-type D2 magnet were measured in order to determine the location of foci where detectors for the physics experiments were to be placed. The focus for trajectories of the same momentum was localized by varying the trajectory start angle at the target. To overcome the problem of instability, which arises when the pulley has to be located behind the focus, an unbalanced pulley was used. Other improvements include methodological features such as the particular design of our air-bearing pulley enabling a "freezing-in" of the wire movement and therefore yielding a very high accuracy of the wire position measurement, the use of a $40 \mu \mathrm{m}$ gold-plated tungsten wire best fulfilling the mechanical and electrical requirements, a fast-regulating power supply in combination with an absolutely calibrated current meter, and the excellent accuracy of modern surveying instrumentation. An overall momentum accuracy of $\Delta p / p \leqslant 10^{-3}$ and a position accuracy of the trajectories of $\pm 1 \mathrm{~mm}$ in the vicinity of the foci have been demonstrated. The precision and accuracy of the floating-wire measurements are discussed and compared with ray-tracing calculations based on measured and calculated field maps. (C) 2001 American Institute of Physics. [DOI: 10.1063/1.1357232]
\end{abstract}

\section{INTRODUCTION}

The ANKE dipole spectrometer facility for the momentum separation of particles ejected from proton-induced reactions at internal targets has recently been installed in the COSY Jülich cooler-synchrotron ring. ${ }^{1}$ The installation consists of three rectangular dipoles, D1, D2, and D3, forming a triangle-like chicane in one of the straight sections of the accelerator ring. The three magnets guide the circulating COSY beam through a thin target in front of D2 and at the same time D2 is used for the separation of ejectiles emitted in a forward direction. One of the experiments performed is the near-threshold production of $\mathrm{K}^{+}$mesons. ${ }^{2}$ They have to be selected from a huge background of protons and $\pi$ mesons. A combination of different particle detectors, called telescopes, placed at the focus surface of the spectrometer magnet solves this task. The required experimental accuracy was to be able to locate the focus position of a certain momentum within $1 \mathrm{~mm}$ where the momentum should be known with $10^{-2}$ relative accuracy. The well-known floating-wire technique (FWT) appeared to be a rather straightforward method to directly determine the necessary foci for the fixed geometry of the $K^{+}$experiment. Other experiments with different geometries of target and detectors ${ }^{1}$ demand a more general knowledge of trajectories through the magnet. Here, numerical ray-tracing techniques

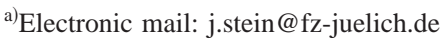

based on field maps have to be applied. The availability of measured and calculated field maps ${ }^{3}$ permitted-as an important crosscheck ${ }^{4}$ - the comparison of the FWT results with trajectories calculated for the same geometry.

Some decades ago, the FWT was commonly applied to determine the magnetic properties of spectrometer and especially accelerator magnets. ${ }^{5-7} \mathrm{~A}$ current-carrying wire freely floating in the magnetic field is balanced by the equilibrium of magnetic force and a mechanical tension force. The tension force is usually produced by running the wire over a balanced low-friction pulley and hanging a weight from it. The wire takes a position representing the trajectories of a charged particle of a certain momentum. The focus formed by trajectories of the same momentum is found by varying the start angle of trajectories at a predefined target point in front of the magnet. Straight trajectories can then be measured in the field-free region outside the magnet. The obtainable accuracy is determined by (i) the friction and hysteresis of the pulley and the wire, (ii) the accuracy of the wire position measurement, and (iii) the stability of the magnetic field and the current in the wire. ${ }^{6}$ Using air-bearing pulleys, accuracies of $\Delta p / p$ from $10^{-3}$ to $10^{-4}$ were reported. ${ }^{8,9}$ The wire remains stable as long as the tension force is applied in front of the focus. Steffen has discussed the instability of the wire when the tension force is applied behind the focus and how to overcome the problem by using, e.g., an unbalanced pulley. ${ }^{5}$

The location of the focus surface at our D2 magnet re- 


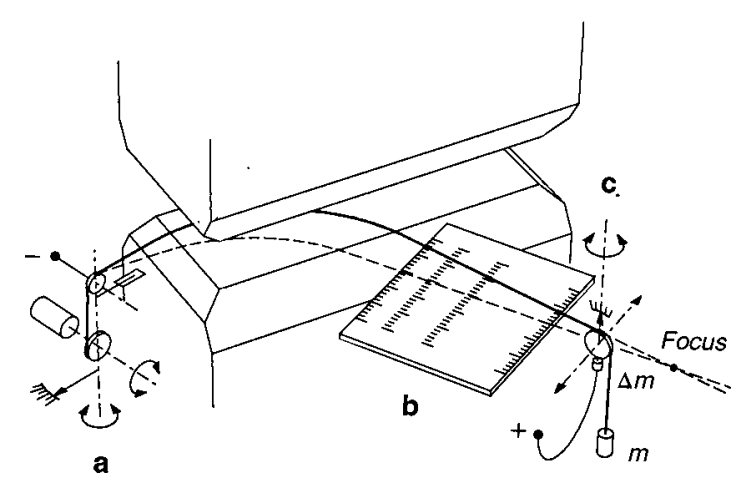

FIG. 1. Principle of the floating-wire setup at the D2 dipole. (a) Wire-length spool mechanism, (b) pulley with pendulum weight $(\Delta m)$ and wire stressing weight $(m)$, and wire-position measuring table with rulers.

quired in some cases placing the wire position measuring device behind the focus. Therefore, it was necessary to study the principle of the unbalanced pulley in detail. The overall momentum accuracy in the physics experiments is expected to be $\Delta p / p \sim 10^{-2}$. In order to be precise enough, our FWT apparatus was devised to have an accuracy of $\Delta p / p$ $\sim 10^{-3}$. We report on the results and on improvements to the FW techniques and methodologies for the D2 spectrometer magnet.

\section{EXPERIMENTAL ARRANGEMENT AND PROCEDURE}

\section{A. The principle of the floating-wire technique}

Figure 1 gives a schematic view of our FWT setup at the D2 magnet illustrating the measurement principle. The D2 magnet is a rectangular C-type magnet with outer pole dimensions of $1400 \times 658 \mathrm{~mm}^{2}$. The pole edges are canted with $45^{\circ}$ and $60^{\circ}$ phases yielding a pole face area of $1240 \times 460$ $\mathrm{mm}^{2}$. The gap height is $200 \mathrm{~mm}$. The starting position of the wire in the midplane of D2 is fixed by a small wheel which is part of a motor-driven spool mechanism to vary the wire length. The whole spool mechanism can be rotated around the vertical axis thereby defining the start angle of the wire. This axis is located at the designated target position $300 \mathrm{~mm}$ in front of the D2 poles and $80 \mathrm{~mm}$ off the longitudinal axis of the magnet. The wire is passed through the magnet gap and hung over the pulley. The tension of the wire is produced by the weight with mass $m$ fixed to the free end. Supplied with a certain current $I$, the wire freely floats in the magnet field following the trajectory of a single-charged particle with a momentum $p$.

The relationship between tension force $F$, current $I$, magnetic field $B$, and momentum $p$ of a particle with charge state $q$ is given by

$$
F / I=B \rho=3.3356 \mathrm{p} / \mathrm{q},
$$

where $\rho$ is the radius of the trajectory curvature in meters and $F, I, B$, and $p$ are in units of $N, A, T$, and $\mathrm{GeV} / \mathrm{c}$, respectively. ${ }^{5,6}$ With $F=g m, m$ in grams, and $g=9.811$ $\mathrm{ms}^{-2}$, the local value (Aachen) of the acceleration of gravity, the momentum $p$ in $\mathrm{MeV} / \mathrm{c}$ is obtained:

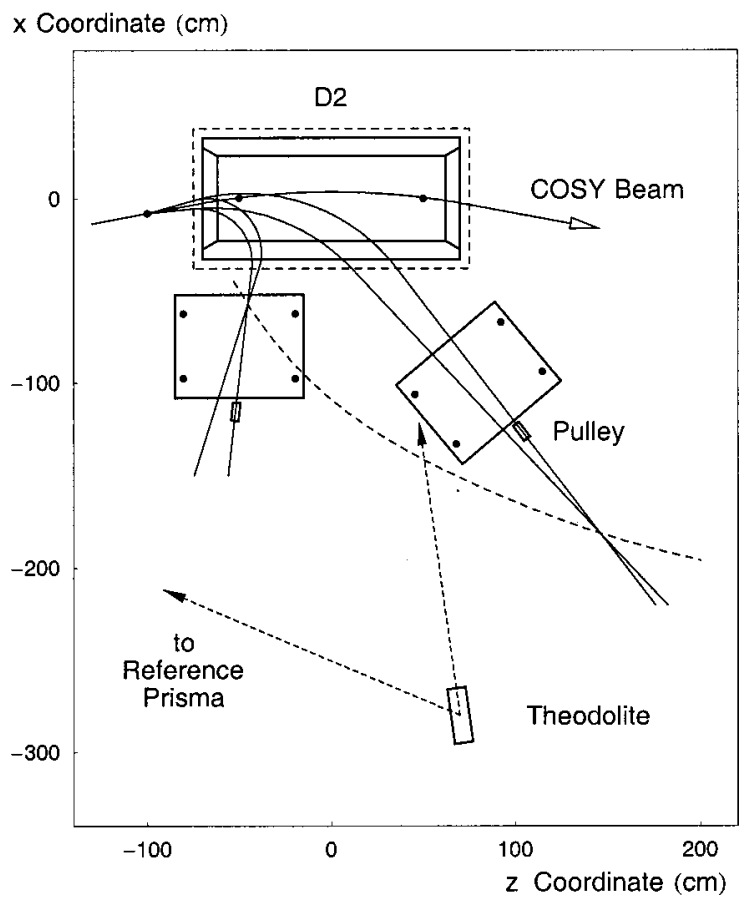

FIG. 2. Floor-plan view of the measuring-table arrangement for a low and a high momentum. For lower momenta the focus is in front of the pulley, for the higher momenta it is behind the pulley. The table position relative to the magnet is determined by a high-precision theodolite.

$$
p=2.9413 \mathrm{~m} / \mathrm{I} \text {. }
$$

The pulley is oriented to the direction of the wire by rotating it around the vertical axis. The position of the trajectory is measured outside the magnetic field by a set of rulers on the measuring table. By varying the wire length and at the same time moving the pulley slightly alongside the measuring table, different start angles can be set. A predefined angle is adjusted when the wire is aligned to the angle control ruler directed towards D2 (Fig. 1). Extrapolation of the trajectories until they cross determines the focal point for the given momentum. Repeating the procedure with different current values and different measuring table positions, the foci for all desired values of momenta are found.

A number of practical difficulties had to be overcome in order to exploit this elegant principle with the desired accuracy. The planned physics experiments with D2 required the determination of trajectories in the momentum range from 140 to $600 \mathrm{MeV} / \mathrm{c}$ at a maximum field of about 1.6 T. Figure 2 illustrates a floor-plan geometry of D2 with the accelerator beam trajectory and trajectories for two momenta, 140 and $500 \mathrm{MeV} / \mathrm{c}$, assuming a hard-edged field of $1.6 \mathrm{~T}$ with an approximate effective length and width of 1500 and $758 \mathrm{~mm}$, respectively, given by the outer dimensions of the poles plus half of the gap height. The expected focus is indicated by the dashed line. Provided the magnetic field is stable and constant during the measurements, the accuracy depends essentially on how accurately the tension force can be determined. An air-bearing design for the pulley is a common technique to reduce friction to a negligible amount. ${ }^{8,9}$ Absolute current measurements of the order of $\Delta I / I=10^{-4}$ can easily be achieved today. 
The challenging problem was the fact that for low momenta, from 140 to $300 \mathrm{MeV} / \mathrm{c}$, the measuring table with the pulley could only be placed behind the focus (Fig. 2). With a well-balanced pulley, the wire is not stable under such conditions. The wire is pulled into the field or drops out of it. ${ }^{5} \mathrm{In}$ the detailed theoretical investigation by Kosodaev ${ }^{10}$ and in the first experimental demonstration by Bounin and Milman ${ }^{11}$ it was shown how the wire can be stabilized by using an unbalanced pulley, for instance, by attaching a small weight $(\Delta m)$ at the periphery of the pulley (Fig. 1). Since the movement of the wire is connected with a turning of the pulley in one direction or the other, the restoring force around the inherent equilibrium position of the pulley prevents the wire from moving away. If the wire length is precisely adjusted such that the pulley, acting as a physical pendulum, moves back to this equilibrium position determined without the wire, the tension force is again well defined. The unbalanced pulley also permitted a "force-free" current connection to the pulley. A piece of $40 \mu \mathrm{m}$ tungsten wire was fixed at the position of the pendulum weight and led in a wide bend to the power supply connector (Fig. 1).

As a consequence of such a setup, the measuring table can always be located at or close to the crossings of the trajectories. The accuracy of the crosspoint determination is appreciably improved in this case, since long-distance extrapolations are avoided. The positions of the measuring table and the target with respect to the magnet were determined by modern surveying techniques applying a specially selected high-precision theodolite ${ }^{12}$ which measures angle and distance at the same time by using prismatic reflectors at the magnet, the measuring table, and the target position (Fig. 2). For the target position measurement the target wheel is replaced by a prismatic reflector.

The choice of the wire is another point of practical importance. In order to cover the whole range of foci, wire lengths from $3.5 \mathrm{~m}$ for $140 \mathrm{MeV} / \mathrm{c}$ to $5.7 \mathrm{~m}$ for $600 \mathrm{MeV} / \mathrm{c}$ had to be used. A gold-plated tungsten wire $40 \mu \mathrm{m}$ in diameter fulfilled all electrical and mechanical requirements. With currents form 0.09 to $0.21 \mathrm{~A}$ and a weight of about $10 \mathrm{~g}$, momenta from 320 to $140 \mathrm{MeV} / \mathrm{c}$ could be defined. A weight of about $20 \mathrm{~g}$ was used for the range from 650 to $280 \mathrm{MeV} / \mathrm{c}$. The use of two different weights was necessary so as not to exceed the safety regulation limit of $60 \mathrm{~V}$ of the current power supply. The resistance of the wire at $0.21 \mathrm{~A}$ was about $90 \Omega / \mathrm{m}$, and the maximum electric power was then not more than $4 \mathrm{~W} / \mathrm{m}$, which kept the temperature of the wire below $100^{\circ} \mathrm{C}$. With weights between 10 and $20 \mathrm{~g}$ sagging of the wire was calculated to be negligible (less than $2.5 \mathrm{~mm}$ ) while the wire was still flexible enough to be manipulated.

\section{B. Pulley design and properties}

The design of our air-bearing pulley fabricated from low-permeability stainless steel is shown in Fig. $3 .{ }^{13}$ Compressed air enters axially into the inner cylinder, is distributed through radial holes into the $0.05 \mathrm{~mm}$ split lifting the outer coaxial cylinder with the wire guiding disk and flows out radially along the gaps formed by the two side disks. The width of the side gaps, about $0.25 \mathrm{~mm}$, is adjustable in order

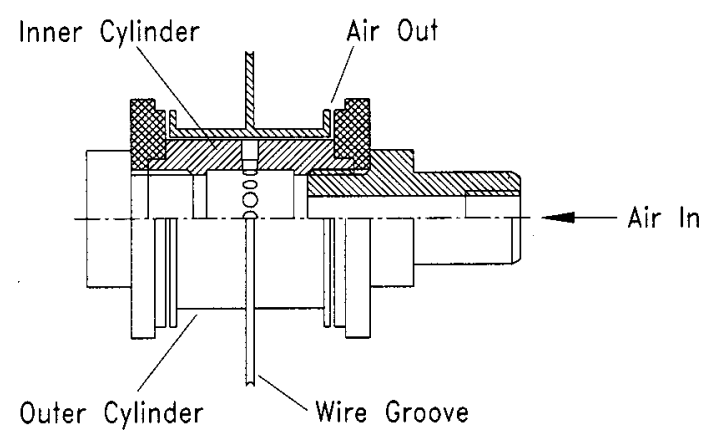

FIG. 3. Cross-sectional view of the air-bearing pulley made of lowpermeability stainless steel. The diameter of the pulley is $60 \mathrm{~mm}$ at the groove for carrying the wire.

to achieve self-stabilization of the outer cylinder with respect to small axial forces. The outer cylinder with the wire guiding disk has a weight of $70 \mathrm{~g}$. The diameter of the groove bottom is $60 \mathrm{~mm}$. The air pressure required to lift the disk is approximately 0.5 bar. Clean dry air from a central compressed-air pipeline was used via a pressure-control valve. During the measurements we applied a pressure of about 1.5 bar. The pulley was fixed to a support which allowed the pulley groove to be aligned with the direction of the floating wire. The support was mounted on a motordriven table so that the pulley was horizontally movable along the measuring table. An integral part of the pulley device was a thin pointer fixed to the wire-guiding disk in order to control the zero position of the unbalanced pulley (see Fig. 1). At the tip of the pointer at a radius of $80 \mathrm{~mm}$ the reading precision was $\pm 0.3^{\circ}$. This corresponds to a wire length variation of $\pm 0.16 \mathrm{~mm}$. The wire spool mechanism was therefore equipped with a precision gear with manual fine control.

The performance of the pulley was investigated with respect to the concentricity between the pulley groove and the surfaces upon which the air acts. Attaching a $200 \mathrm{mg}$ weight of the periphery of the wire guiding disk, the well-balanced pulley was transformed into a physical pendulum. Using the $40 \mu \mathrm{m}$ tungsten wire, two $20 \mathrm{~g}$ weights were suspended to the left and right of the pulley. Adding additional small weights of some milligrams on one or the other $20 \mathrm{~g}$ weight, left and right displacement angles of the pendulum were measured over a range of about $\pm 45^{\circ}$ as a function of the additional weights. This procedure was repeated for the other three sectors of the disk. The deviations from the ideal displacement function indicated the inhomogeneities of the concentricity. Deviations of up to $60 \mu \mathrm{m}$ radius variations were found. However, a $90^{\circ}$ wide region could be selected where the radius was constant within $20 \mu \mathrm{m}$. This region was used for further application. The value of the radius variation in the selected sector was confirmed by observing wire displacements through a measuring microscope (magnification 30 ) while turning the pulley over a range of $150^{\circ}$. In addition, the pulley revealed a slight intrinsic moment of rotation caused by the air flow in the split which was found to be about $(10 \pm 5) \mathrm{mg}$ equivalent load. This intrinsic moment of rotation, referred to as the "turbine effect," only has to be considered for the balanced mode. In the unbalanced mode it 
is compensated by the pendulum action. The uncertainty of $\pm 5 \mathrm{mg}$ stems from air-flow-induced fluctuations which can also be interpreted as friction. This uncertainty is included in the reading error of the equilibrium position.

\section{Operational procedure}

The pendulum weight necessary for stabilizing the wire if the pulley is placed behind a focus can be estimated following the theory of Kosodaev. ${ }^{10}$ The weight should be as light as possible so that the uncertainty of the pointer reading does not contribute too much to the uncertainty of the wire tension. However, a system close to instability becomes impractical due to the low oscillation frequency around the equilibrium state. A weight of $\Delta m=0.8 \mathrm{~g}$ for the $10 \mathrm{~g}$ load $(1.2 \mathrm{~g}$ for $20 \mathrm{~g})$ attached at the periphery of the pulley was found to be appropriate. In these cases the oscillation frequency of the pulley was acceptable $(\approx 0.5 \mathrm{~Hz})$. The pointer reading uncertainty was about the same as the uncertainty caused by the turbine effect.

The principle of the unbalanced pulley enabled a specific experimental procedure to be applied which turned out to be extremely advantageous. Cutting the compressed-air flow allowed the oscillating pulley to be stopped exactly in the equilibrium state. In this "frozen state" the reading of the wire position at the table was highly reproducible. At the beginning of the investigations, additional slow movements of the wire were observed due to fluctuating convective heat losses from the hot wire. This effect was avoided by reducing the regulation time constant of the wire current power supply.

To find the equilibrium of the wire in the unbalanced mode is slightly more complicated since the wire length is no longer a free parameter. Wire length and position of the pulley have to be adjusted alternately until the pulley pointer is back in its zero position under the condition of a welldefined wire start angle. A typical time needed for this operation was $15 \mathrm{~min}$ so that a full set of measurements for one momentum could be performed in about $2 \mathrm{~h}$. Nevertheless, comparing the main error sources of the balanced and unbalanced mode, we would always prefer the unbalanced mode because it avoids the extrapolation of trajectories which introduces random errors in wire position readings at the measuring table. The main random error in the unbalanced mode - the uncertainty of the pulley pointer reading — can be kept small compared to the other errors (see Table I, Sec. IV).

\section{Wire-position determination}

The position of a wire stretched over the measuring table was measured by a set of four $0.5 \mathrm{~m}$ long rulers with $0.5 \mathrm{~mm}$ scaling mounted on a $700 \times 560 \mathrm{~mm}^{2} \times 20$-mm-thick aluminum plate. The rulers were mounted on the table at relative positions of $0,101.1,250.5$, and $535.1 \mathrm{~mm}$. Mirrors below the rulers enabled a parallax-free reading. The height of the measuring table was adjusted such that the wire passed 3-4 $\mathrm{mm}$ above the rulers ensuring good visibility of the wire and its image. Placing a lens on a glass plate about $100 \mathrm{~mm}$
TABLE I. List of the various error sources influencing momentum and position of measured FWT trajectories.

\begin{tabular}{|c|c|c|}
\hline Error source & & Value \\
\hline \multicolumn{3}{|l|}{ Relative random errors of momentum } \\
\hline Dispersion of current readings & $(\Delta I / I)_{\text {meas }}$ & $\pm 1 \times 10^{-4}$ \\
\hline Dispersion of tension force & $(\Delta F / F)_{\text {pend }}$ & $\pm 3 \times 10^{-4}$ \\
\hline Resulting momentum error (rms) & $(\Delta p / p)_{\text {random }} \approx$ & $\pm 3 \times 10^{-4}$ \\
\hline \multicolumn{3}{|l|}{ Absolute geometrical errors } \\
\hline$\overline{\text { Wire position reading on rulers }}$ & & $\pm 0.1 \mathrm{~mm}$ \\
\hline Positioning and alignment of rulers & & $\pm 0.2 \mathrm{~mm}$ \\
\hline Table position relative to the magnet & & $\pm 0.5 \mathrm{~mm}$ \\
\hline Table orientation relative to the magnet & & $\pm 2 \mathrm{mrad}$ \\
\hline Dispersion in settings of start angles & & $\pm 4 \mathrm{mrad}$ \\
\hline \multicolumn{3}{|l|}{ Relative systematic errors of momentum } \\
\hline Mass of the main weight & $\Delta M / M$ & $\pm 1 \times 10^{-5}$ \\
\hline Acceleration of gravity & $\Delta g / g$ & $\pm 2 \times 10^{-5}$ \\
\hline Calibration of the current meter & $(\Delta I / I)_{\mathrm{cal}}$ & $\pm 2 \times 10^{-4}$ \\
\hline Radius of the pulley & $\Delta R / R$ & $\pm 6 \times 10^{-4}$ \\
\hline Drift of the magnetic field & $\Delta B / B$ & $\pm 2 \times 10^{-4}$ \\
\hline Resulting momentum error (rms) & $(\Delta p / p)_{\mathrm{sys}} \approx$ & $\pm 7 \times 10^{-4}$ \\
\hline
\end{tabular}

above the table and illuminating the wire from the side made ruler readings with a precision of $\pm 0.1 \mathrm{~mm}$ possible.

The table plate was made of a special stress-free $\mathrm{Al}$ alloy. It was accurately machined to be flat within $0.1 \mathrm{~mm}$. Four precisely machined boreholes to accommodate the prismatic reflectors were arranged in a rectangle with lateral lengths of $610 \pm 0.05$ and $350 \pm 0.05 \mathrm{~mm}$ (Fig. 2). The coordinates of these four prism positions together with two magnet positions and the target position were determined for each separate table location. The basic coordinate system of a measurement is formed by the position of the theodolite and a reference prism placed in the prolongation of the magnet axis. Applying appropriate coordinate transformations, the wire positions on the table are finally transferred into the D2 reference coordinate system with its origin at the center of the magnet (Fig. 2). A least-squares fit to the four points obtained for each trajectory was used in order to obtain information about the straightness of the trajectories and about uncertainties of their position and direction.

The target position only $300 \mathrm{~mm}$ away from the iron edge is already located in the fringe field. In order to provide a well-specified start angle, the curvature of the trajectories was taken into account. The deviation of the wire from a straight trajectory $150 \mathrm{~mm}$ in front of the target point was theoretically determined and checked by a $1 \mathrm{~mm}$ parallax-free mirror scale mounted on the angle control ruler (see Fig. 1).

\section{RAY-TRACING CALCULATIONS BASED ON FIELD MAPS}

The ANKE dipoles D2 and D1/3 were designed on the basis of three-dimensional field map calculations. The MAFIA code $^{14}$ has turned out to be the most appropriate tool. ${ }^{3}$ In order to verify the theoretical results, D2 was experimentally investigated using an appropriate field-mapping machine. ${ }^{15}$ In the physics experiments, ray tracing is a necessary prerequisite for momentum reconstruction. The GEANT $\operatorname{code}^{16}$ is 


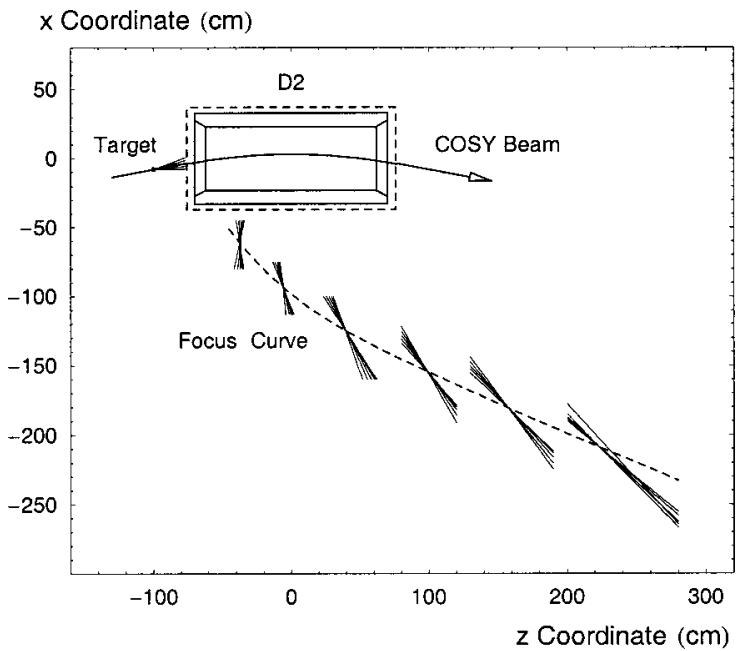

FIG. 4. Measured floating-wire crossings for the six momenta 140.4, 207.4, 291.3, 400.3, 500.3, and 600.1 MeV/c forming the midplane focus curve for the given target position $(z=-99.67 \mathrm{~cm}$ and $x=-7.86 \mathrm{~cm})$. The dashed line around the magnet represents the effective hard-edged field boundary.

used as a powerful tool to cover all aspects of the detection process. The comparison of calculated trajectories based on field maps with those directly determined by FWT measurements will give the necessary confidence in the correctness of the momentum reconstruction procedure.

For this article, the MATHEMATICA ${ }^{17}$ program package was used to calculate the trajectories. It provides features such as interpolation of field maps and numerical solutions for differential equations. The equation of motion for charged particles in the D2-midplane magnetic field reads

$$
x^{\prime \prime}+\frac{B(z, x)}{B \rho}\left[\left(1+x^{\prime}\right)^{2}\right]^{3 / 2}=0,
$$

where $B(z, x)$ is the local magnetic field value taken from the field map by interpolation and $B \rho=3.3356 p$ is the magnetic rigidity $(\mathrm{Tm})$ of a particle with momentum $p(\mathrm{GeV} / \mathrm{c})$. The measured and calculated field maps are given in a $20 \times 20$ $\mathrm{mm}^{2}$ grid. Since the measured map in the horizontal plane covers only $80 \%$ of the range necessary for ejectile trajectories of interest, the measured map was complemented by the calculated map. ${ }^{3}$ The numerical accuracy of the MATHEMATICA calculations was checked by tracing a hypothetical trajectory in the forward direction and, using the result obtained, back to the target. A maximum deviation of $0.2 \mathrm{~mm}$ from the starting point is only seen in the longitudinal direction for low-momentum trajectories where the bending angle is large.

During the FWT measurements the characteristic maximum field value $B_{\max }$ at the center of the magnet was checked by a Hall probe. A nuclear magnetic resonance probe was used to calibrate $B_{\max }$ as a function of the supply current over the whole current range. The measured value for $100 \%$ current was $1.573 \mathrm{~T}$.

\section{EXPERIMENTAL RESULTS AND ERROR DISCUSSION}

Figure 4 shows an overview of the results of the FWT measurements. For each of the six momenta, 140.4, 207.4,
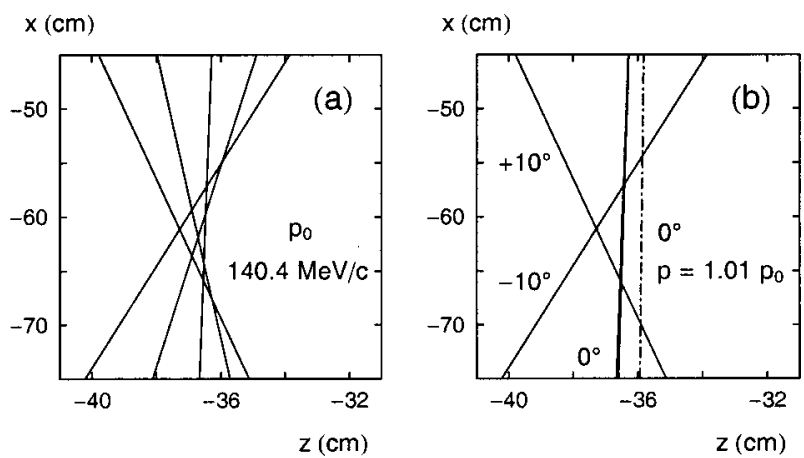

FIG. 5. Demonstration of the reproducibility of the FWT measurements. (a) Five $140.4 \mathrm{MeV} / \mathrm{c}$ trajectories for different start angles. (b) The apparently thicker line represents two $0^{\circ}$ trajectories measured on different days. As an indicator of the momentum resolution, the dot-dashed line was determined with $1 \%$ higher momentum.

291.3, 400.3, 500.3, and 600.1 MeV/c, five trajectories were traced with start angles of $-10^{\circ},-5^{\circ}, 0^{\circ},+5^{\circ},+10^{\circ}$ relative to an assumed accelerator beam angle of $+10.6^{\circ}$. For the first three momenta the unbalanced pulley was applied, for the other three the balanced one. The momenta were derived according to Eq. (2) where the value of the mass $m$ of the weights used $(9.9847 \mathrm{~g}$ in the range $140-320 \mathrm{MeV} / \mathrm{c}$ and $19.9233 \mathrm{~g}$ in the range $280-650 \mathrm{MeV} / \mathrm{c}$ ) was modified by a few systematic corrections. Expressed in equivalent mass, these were $+(10 \pm 1) \mathrm{mg}$ for the piece of wire between the pulley and weight, $-(10 \pm 5) \mathrm{mg}$ for the turbine effect (only during measurements with the balanced pulley) and $+(7$ to $15 \pm 3) \mathrm{mg}$ for small magnetic forces acting on the current supply wire when the measuring table was close to the magnet (for 140.4, 207.4, 400.3, and 500.3 MeV/c). Sagging of the wire may influence the balance of forces and may cause a deviation of the trajectory from the midplane of the magnet where the vertical component of the field strength is lower. In the worst case at a free length of $4 \mathrm{~m}$ sagging was $2.5 \mathrm{~mm}$, which is negligible. The geometrical conditions for the measurement were chosen such that they represent a typical application in the planned physics experiments. (Maximum magnetic field, target at a nominal position of $z=-100 \mathrm{~cm}$ and $x=-8 \mathrm{~cm}$.) The measured target position $(z=-99.67$ $\mathrm{cm}$ and $x=-7.86 \mathrm{~cm}$ ) was close to the nominal position. The dashed line in Fig. 4 represents the midplane focus curve for the applied maximum field of $1.573 \mathrm{~T}$.

Aberration is not resolved in the global view of Fig. 4. Only the $+10^{\circ}$ trajectory for $600.1 \mathrm{MeV} / \mathrm{c}$ does not hit the focal point formed by the other trajectories. A magnified view on the focal area clearly resolves aberration. As an example, the aberration pattern for $140.4 \mathrm{MeV} / \mathrm{c}$ trajectories is shown in Fig. 5(a). Here, the pulley was operated in the unbalanced mode. The trajectories near the focus were located at the measuring table yielding the highest possible precision of trajectory position readings. As a check for the overall reproducibility a second $0^{\circ}$ trajectory was determined once more after the whole apparatus had been shut down and switched on again the next day. Figure 5(b) indicates these two $0^{\circ}$ trajectories by a slightly thicker line. A second $0^{\circ}$ trajectory determined with a $1 \%$ higher momentum clearly demonstrates a reproducibility of $\sim 10^{-3}$. It should be noted 
that before starting a measurement under new conditions the equipment had a $1 \mathrm{~h}$ warm up.

In order to comment on the overall accuracy it is necessary to study the various error sources in detail. Two kinds of errors can be distinguished: errors involved in the momentum determination and errors influencing the accuracy of the wire position determination. Random errors of wire current and tension force may occur within one set of measurements for a certain momentum and in measurement sets for different momenta. Once the wire is fixed in an equilibrium, we may have dispersion of current readings and dispersion of the tension force (see Table I). The error sources are mutually independent and, when added quadratically, yield a random error of $(\Delta p / p)_{\text {random }} \approx \pm 3 \times 10^{-4}$. The dispersion of the tension force is caused by the uncertainty of the pulley zero point, $\pm 0.3^{\circ}$, comprising fluctuations of the intrinsic moment of rotation, friction, and reading errors. The random error holds within a set of measurements for one momentum as well as for sets of different momenta. The second kind of error is purely of geometrical origin comprising the position and orientation of the rulers on the measuring table as well as position and orientation of the latter relative to the magnet. Finally, there are systematic errors which are the same in all measurements (Table I). They are also mutually independent and, added quadratically, yield $(\Delta p / p)_{\text {sys }} \approx \pm 7 \times 10^{-4}$.

The geometrical errors essentially depend on the distances between crosspoints and the closest ruler. These errors are minimized if the trajectories cross at the measuring table as is the case in the unbalanced mode. This error depends on the accuracy and precision of the theodolite. The manufacturer gives values of $0.001 \mathrm{mrad}$ for the angle and $0.5 \mathrm{~mm}$ for the distances. The precisely machined distances of the drilled holes in the table plate yielded a direct measure of the accuracy of the theodolite. Ten measurements of these distances for five different table locations resulted in mean values of $610.11 \pm 0.04$ standard error and $350.10 \pm 0.05 \mathrm{~mm}$ standard error with a maximum deviation of $0.25 \mathrm{~mm}$ compared to 350 and $0.17 \mathrm{~mm}$ compared to $610 \mathrm{~mm}$. The $1 \mathrm{~m}$ distance between the two marks on the magnet was always accurate within $\pm 0.05 \mathrm{~mm}$. These numbers reveal the high performance of modern surveying instruments and ensure that the absolute locations of trajectory crosspoints relative to the magnet are accurate within $\pm 0.5 \mathrm{~mm}$. Due to the extreme angle accuracy, the orientation error of the measuring table is assumed to be smaller than 2 mrad.

The measured field maps ${ }^{3}$ enabled the assumptions to be checked for the various errors of the FWT data. In Fig. 6 measured and calculated trajectories for 291.3 and 500.3 $\mathrm{MeV} / \mathrm{c}$ are compared in the focus region in a magnified view. The 291.3 MeV/c trajectories were determined with the unbalanced pulley, and the $500.3 \mathrm{MeV} / \mathrm{c}$ trajectories in the balanced mode. The agreement with the calculated trajectories is almost perfect. The dot-dashed line refers to $1 \%$ higher momentum. The difference between measured and calculated trajectories corresponds to $\Delta p / p \leqslant 0.5 \times 10^{-3}$.

It is interesting to discuss the shape of the aberration pattern in view of start angle errors. The five trajectories in Fig. 5 form a kind of triangle with a hyperbolically shaped "hypotenuse." As shown in Fig. 7, this line (the caustic) is
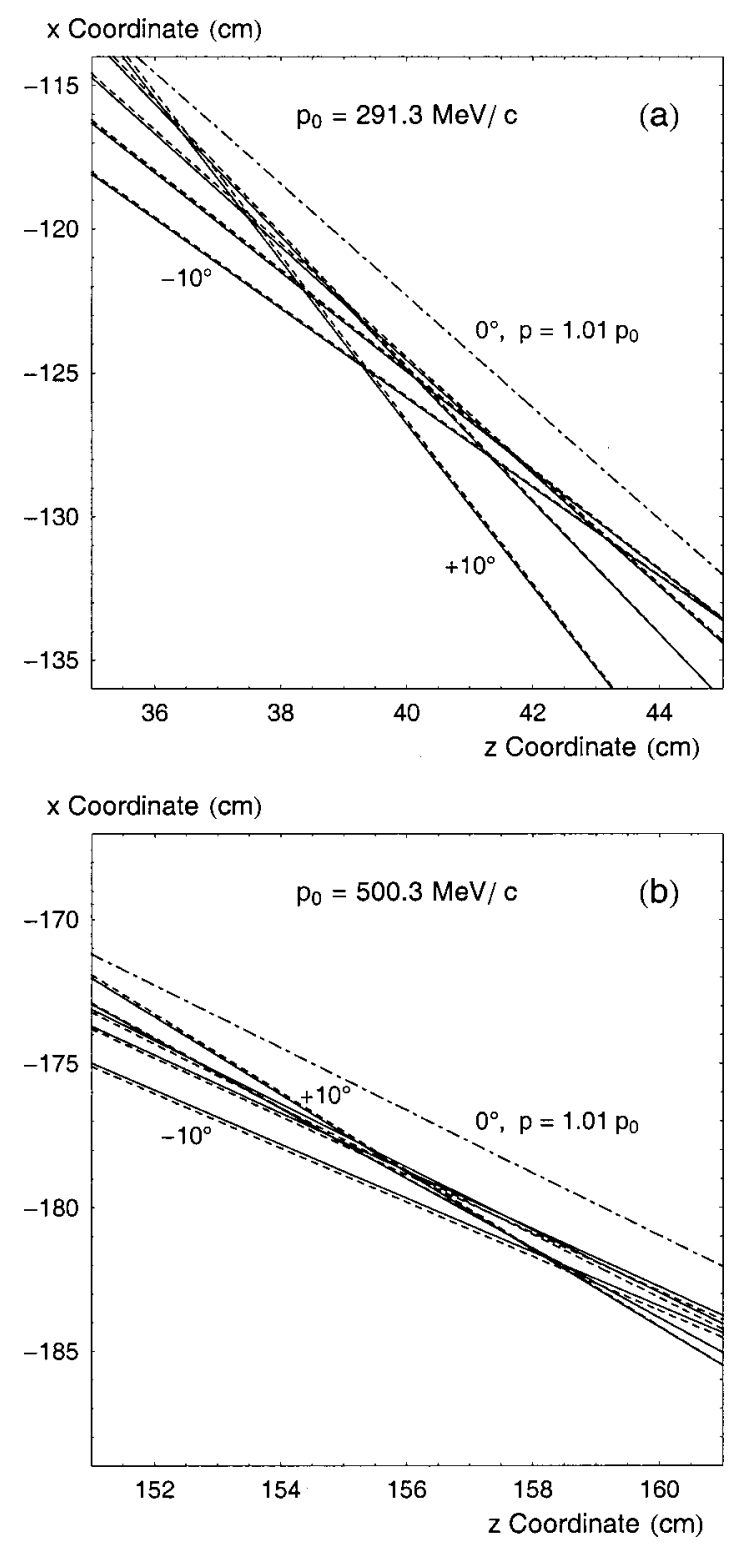

FIG. 6. Comparison of measured trajectories with field-map-based calculated trajectories for two selected cases: (a) unbalanced pulley, (b) balanced pulley. The agreement in the focal region is in the order of $\Delta p / p=10^{-3}$ as indicated by $0^{\circ}$ trajectories measured with a $1 \%$ higher momentum.

independent of small input angle deviations. It is formed by the foci of a series of trajectories of adjacent start angles. At the tip of the aberration triangle, however, dispersion of the start angles leads to increasing uncertainty. Using the crosspoint of trajectories in the limit of small angles with respect to $0^{\circ}$ enables the unique definition of the focal point. In our case, the crossings of $+5^{\circ}$ and $-5^{\circ}$ trajectories are precise enough for positioning of the detectors.

Tracing the trajectories backwards, starting with the measured trajectories in their focus region, provides another way of assessing the measuring errors. Here, the start angle error is not involved since all trajectories originate from the target point. Figure 8 shows such backward trajectories for 291.3 and $500.3 \mathrm{MeV} / \mathrm{c}$ in the vicinity of the target. The trajectories meet almost perfectly in one point indicating very small random errors within a group of measurements at 


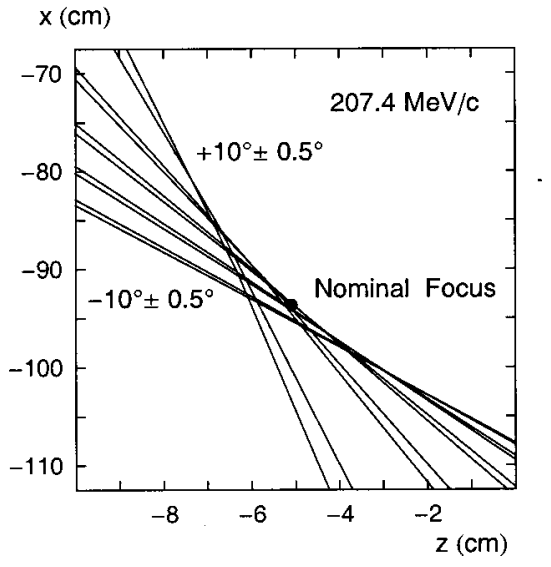

FIG. 7. The crosspoints of adjacent trajectories with slightly different start angles form the caustic of the aberration, here shown for 207.4 MeV/c. The caustic is insensitive to start-angle errors. The nominal focus can be defined by the crossing in the limit of small angles.

the same momentum. For instance, a $\Delta p / p=+0.3 \times 10^{-3}$ would move the $500.3 \mathrm{MeV} / \mathrm{c}+5^{\circ}$ trajectory such that it would cross the other trajectories just as optimally as in the 291.3 MeV/c case. However, a clear displacement from the measured target position is evident, which may be due to several systematic errors such as overall momentum errors, and/or table position errors. It should be noted that lack of mechanical precision in mounting the prismatic reflector on the axis of the target rotation mechanism might have been an additional cause. An estimate of the target rotation error is shown as an ellipse around the measured target position. The small rectangle describes the dispersion of theodolite readings.

Figure 9(a) shows all the trajectories calculated backwards in the momentum range from 207.4 to $600.1 \mathrm{MeV} / \mathrm{c}$. All the trajectories meet close to the measured target point forming a waist of $2 \mathrm{~mm}$ width. Compared to the measured target position the waist seems longitudinally shifted by about $-2 \mathrm{~mm}$. Figure 9(b) shows the ensemble of calculated trajectories superimposed by the various error contributions as discussed $\left(\Delta p / p= \pm 0.07 \%, \quad \Delta_{\text {position }}= \pm 1.0 \mathrm{~mm}\right.$, $\left.\Delta_{\text {orientation }}= \pm 2.0 \mathrm{mrad}\right)$. Position and orientation comprise
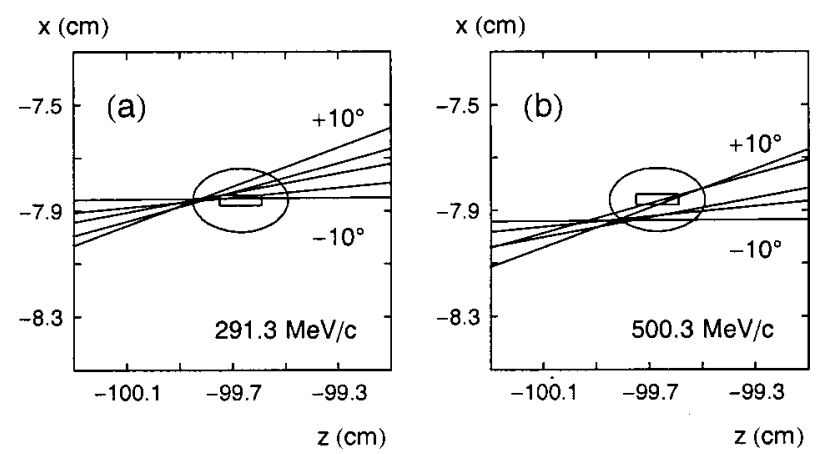

FIG. 8. Trajectories calculated backwards in the vicinity of the target. (a) The $291.3 \mathrm{MeV} / \mathrm{c}$ trajectories cross rather perfectly at a point. (b) The 500.3 group indicates small random errors. The general shift of the crossing regions from the measured target position (the rectangle describes the dispersion of theodolite readings) is caused by systematic errors of momentum, orientation, and the target rotation (shown by the surrounding error ellipse).
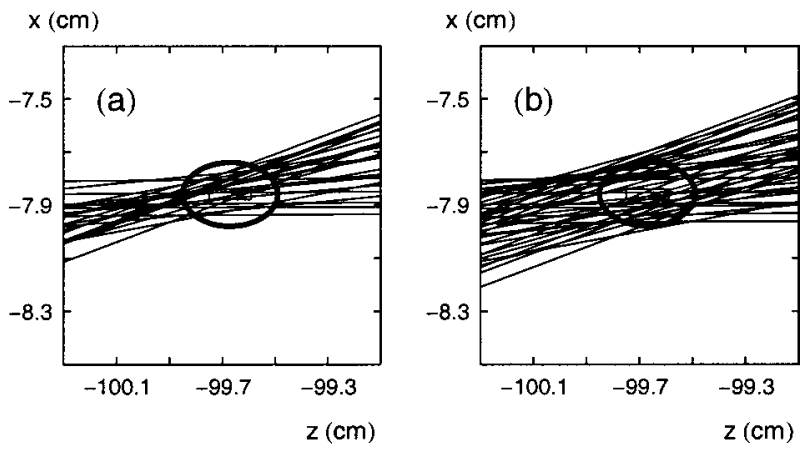

FIG. 9. (a) Superposition of measured trajectories calculated backwards in the vicinity of the target. (b) The same trajectories superimposed by the assumed measuring errors for momentum, position and orientation demonstrating the overall measurement accuracy of $\Delta p / p \leqslant 10^{-3}$.

the least-squares errors of trajectories and the assumed errors for the measuring table. The comparison clearly demonstrates that the experimental estimates concerning the errors are correct. The overall results shown in Fig. 9 confirm the precision and the accuracy of the FWT as well as the reliability of the field mapping measurements. It is interesting to note that the applied $B_{\max }=1.573 \mathrm{~T}$ fits well with the FWT results and that the pure MAFIA field, with its $B_{\max }$ normalized to $1.573 \mathrm{~T}$, results in a deviation equivalent to a $0.15 \%$ too high $\int B d l$ product. ${ }^{3}$ The availability of an independent method to experimentally determine charged particle trajectories for the ANKE magnet D2 resolved a number of questions about the ray-tracing calculations. It was also shown that modern field map codes like the three-dimensional MAFIA code may deliver results close to the original accuracy goal of $\Delta p / p \approx 10^{-3}$. A double check with a method like the FWT may then provide the desired confidence. ${ }^{4}$

\section{ACKNOWLEDGMENTS}

The authors would like to thank Professor Emeritus O. W. B. Schult for valuable advice and his continuous interest in this work. This work was supported in part by the Russian Ministry of Sciences, Deutsche Forschungsgemeinschaft Grant No. 436 RUS 113/430, and Bundesministerium für Bildung und Forschung Grant No. RUS-649-96.

${ }^{1}$ S. Barsov et al., Nucl. Instrum. Methods Phys. Res. (to be published).

${ }^{2}$ S. Barsov et al., Nucl. Phys. A 675, 230 (2000).

${ }^{3} \mathrm{M}$. Büscher et al. (unpublished).

${ }^{4} \mathrm{G}$. Moritz, in Measurement and Alignment of Accelerator and Detector Magnets (CERN Accelerator School, Anacapri, Italy, 1997, CERN 98-05, 1998), p. 251

${ }^{5}$ K. G. Steffen, High-Energy Beam Optics (Interscience, New York, 1965), and references therein

${ }^{6}$ Uriel Vogel, Rev. Sci. Instrum. 36, 188 (1965).

${ }^{7}$ Z. F. Wang, S. J. Greene, and Michael A. Plum, Nucl. Instrum. Methods Phys. Res. A 234, 413 (1986).

${ }^{8} \mathrm{H}$. Bichsel, in Proceedings of the 1965 Symposium on Magnet Technology, edited by H. Brechna and H. S. Gordon (Stanford University, Stanford, CONF-650922), p. 467.

${ }^{9}$ V. R. W. Edwards and A. E. Osborne, Rev. Sci. Instrum. 38, 806 (1967).

${ }^{10}$ M. S. Kosodaev, Nucl. Instrum. Methods 133, 143 (1976).

${ }^{11}$ P. Bounin and P. Milman, Rev. Sci. Instrum. 34, 1448 (1963). 
${ }^{12}$ Wild, Tachymeter Model TC2002, marketed by Leica, Switzerland.

${ }^{13}$ A. I. Alexeev and S. L. Belostocky, Preprint PTI-265, Leningrad, 1967 (unpublished).

${ }^{14}$ Computer code MAFIA, MAFIA-4, set http://www.cst.de
${ }^{15}$ G. Moritz et al., IEEE Trans. Magn. 30, 2650 (1994).

${ }^{16} \mathrm{~S}$. Giani et al., computer code GEANT, (CERN, October 1994).

${ }^{17} \mathrm{~S}$. Wolfram, The Mathematica Book, 4th ed. (Cambridge University Press, Cambridge, 1999). 